\title{
An Operator Based Modeling and Compensation of VCM Actuator Pivot Friction in a 1.8-inch HDD *
}

\author{
C. Du* L. Xie ${ }^{* *}$ J. Zhang* \\ * A ${ }^{*}$ STAR, Data Storage Institute(DSI), 5 Engineering Drive 1, \\ Singapore 117608 (e-mail: DU_Chunling@dsi.a-star.edu.sg). \\ ** School of Electrical and Electronic Engineering, Nanyang \\ Technological University, Nanyang Avenue, Singapore 639798 (e-mail: \\ elhxie@ntu.edu.sg)
}

\begin{abstract}
The pivot friction of voice-coil-motor (VCM) actuator is measured for a 1.8-inch small disk drive with disk rotating and slider flying. The measurement is carried out under the conditions that the actuator is controlled and the head movement amplitude is growing by changing the references. The hysteresis of friction versus head position is then obtained. An operator based modeling approach is adopted for the hysteresis, and an optimal model is obtained by minimizing the energy gain between the head position and the modeling error. It is also found that the frequency response of the actuator model with the inclusion of the hyteresis model matches well with the measured frequency response of the actuator. A friction compensation method based on the nonlinear hysteresis model is then proposed. The simulation and implementation results demonstrate a significant improvement in disturbance rejection in low frequency range.
\end{abstract}

\section{INTRODUCTION}

The demand for high capacity hard disk drives (HDDs) is growing continuously [1]. So far, the increased capacity has been mainly achieved by enhancing recording density. Larger areal density requires the head positioning system in HDDs using voice-coil-motor (VCM) actuators to have a very precise positioning accuracy. Friction in the actuator pivot [2] [3] is known to limit the low frequency gain of the servo loop. Translated to the error rejection function or sensitivity function, it lifts the magnitude of the sensitivity function at low frequency [4], and thus reduces the ability of the servo loop to reject vibrations at low frequency and affects the positioning accuracy of the read-write head. On the other hand, the trend towards smaller form factor is prosperous in the magnetic disk storage industry, as hard disk drives enter mobile applications such as portable music players, portable photo/video viewers, and automotive systems [9]. Compared with that in larger form factor disk drives, the contribution of the friction to servo performance is increased in a small disk drive due to its small actuator inertia.

In literature there are basically two kinds of methodologies for friction control: Model-based friction compensation and non-model based friction control. For friction model, it can be roughly classified into three categories: static model, dynamic model and friction observer. There are various static friction models, for example, coulomb friction model, viscous friction model, negative viscous friction model, friction models with stribeck effect, etc. However, static friction models cannot capture observed friction phenomena like the hysteresis phenomena, position

\footnotetext{
‡ This paper is supported by A*Star SERC Grant No. 0521010036.
}

dependence, variations in the breakaway forces, etc. Therefore, friction models involving dynamics are necessary to describe the friction phenomena accurately. [4] considers a new model-based friction compensator for servo control of HDDs. By feeding back the model-based estimated friction forces into the VCM actuator, the dynamics between the read/write head position and the VCM current can be linearized. However, the friction model needs to be iteratively improved and verified. Preload and two-slope model is used to model friction in the time domain [5] and the frequency domain [3] with different input excitation levels. While the time-domain approach provides a good match between the time domain response of the model and the data collected, it cannot guarantee a good match in frequency domain, and vice versa. Paper [10] presents modeling and compensation methods for flex cable bias in a micro HDD, and the design of the HDD servo system utilizes an enhanced nonlinear control technique. Note that the measurement of nonlinearity is carried out with the disk being stationary.

On the other hand, non-model based approaches such as the neural network method [7][8] and disturbance observer method [6] are proposed. The neural network method does not require to fully know the nonlinearity model, but its implementation in real disk drives seems difficult. In [6], Ishikawa and Tomizuka proposed a novel method for the cancellation of pivot nonlinearities. The method consists of an accelerometer and a disturbance observer. The accelerometer is employed to linearize the dynamics from the desired input signal to carriage angular acceleration, and the observer estimates the nonlinear disturbances due to pivot friction for disturbance cancellation. 
In this paper VCM actuator pivot friction is measured for a 1.8-inch small disk drive with disk rotating and slider flying. An operator based model is used to model the friction and optimal model parameters are obtained by minimizing the energy gain between the actuator position and the modeling error. With the inclusion of the friction model, the frequency response of the actuator model also fits well to the measured frequency response of the actuator. Based on the resultant friction model, the nonlinear friction is compensated by injecting the friction force into the VCM actuator. Simulation and experimental results clearly demonstrate the effectiveness of the proposed method.

\section{FRICTION MEASUREMENT IN A 1.8-INCH HDD}

The HGST 1.8-inch small drive with $4200 \mathrm{rpm}$ rotational speed is dismantled and the friction of the VCM actuator will be measured via laser Doppler vibrometer (LDV). The LDV displacement range is $0.5 \mu \mathrm{m} / \mathrm{V}$. The frequency response of the VCM actuator is measured with swept sinewave via Dynamic Signal Analyzer (DSA) and shown in Fig. 1.
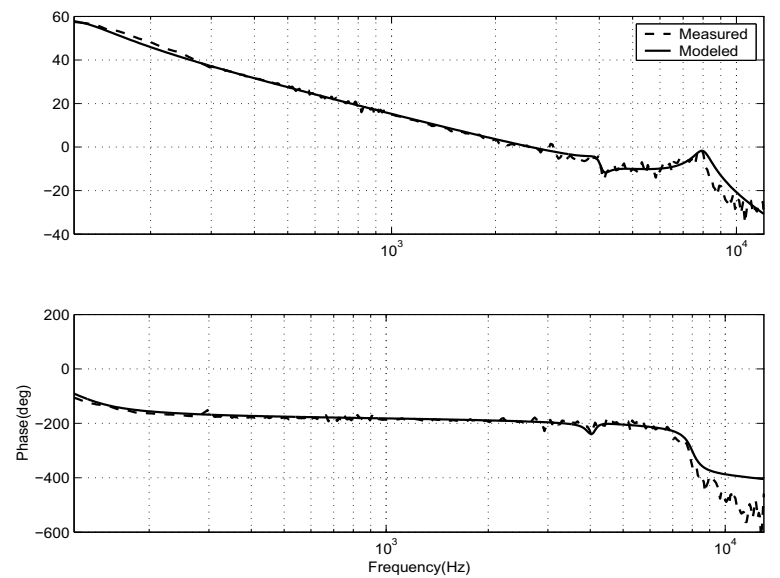

Fig. 1. One measured frequency response of the VCM actuator.

Due to the fluctuation of the head when the disk is rotating, it is difficult to have a steady displacement signal of the head. Thus the friction measurement is carried out under the closed loop as shown in Fig. 2 with the following controller $C(z)$. The sampling time $T_{s}$ is $83.3 \mathrm{~ms}$. With the controller, the open loop $0 \mathrm{~dB}$ crossover frequency is 945 $\mathrm{Hz}$, the gain margin is $8.7 \mathrm{~dB}$, and the phase margin 49 deg. In the rest of the paper, all measurements are carried out in the closed loop system.

$$
\begin{gathered}
C(z)=k_{c}\left(k_{p}+k_{d} \frac{z-1}{T_{s} z}+k_{i} \frac{T_{s}}{z-1}\right) \\
\times \frac{0.9023 z^{2}+0.9467 z+0.7242}{z^{2}+0.929 z+0.6442}, \\
k_{c}=0.0625, k_{p}=0.8, k_{d}=400 e-6, k_{i}=400 .
\end{gathered}
$$

A $10 \mathrm{~Hz}$ sinusoidal signal with increasing amplitude of $0.5,1$, and $3 \mathrm{~V}$ is respectively used as the reference signal in Fig. 2. The control signal $u$ and displacement $x$ are measured, and shown in Fig. 3.

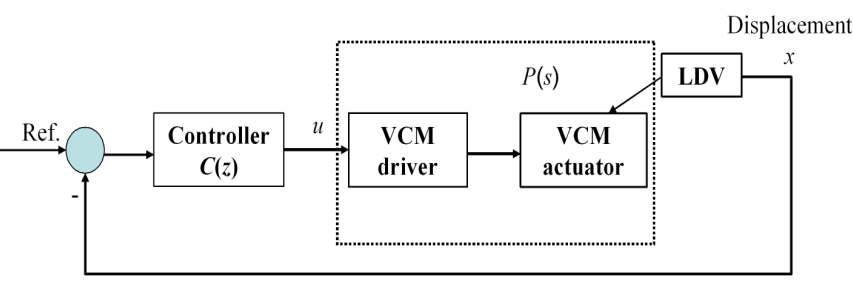

Fig. 2. Closed control loop of a disk dive with a VCM actuator for friction measurement via LDV.

The VCM actuator model with the consideration of nonlinearity $F(x)$ is shown in Fig. 4, which includes two pure integrators, the resonance modes $P_{r e s}(s)$ and the gain $K$. $P_{\text {res }}(s)$ and $K$ can be determined based on Fig. 1 and given by

$$
\begin{aligned}
& P_{\text {res }}(s)= \\
& \frac{s^{2}+1081 s+7.3 \times 10^{8}}{\left(s^{2}+1056 s+6.964 \times 10^{8}\right)\left(s^{2}+6032 s+2.527 \times 10^{9}\right)} \\
& K=1.2052 \times 10^{17} .
\end{aligned}
$$

With the measured $u$ and $x, u_{a}$ can be obtained from $x$, and thus $f=u-u_{a}$. The relation between $x$ and $f$ can be obtained and shown as hysteresis curves in Fig. 7.

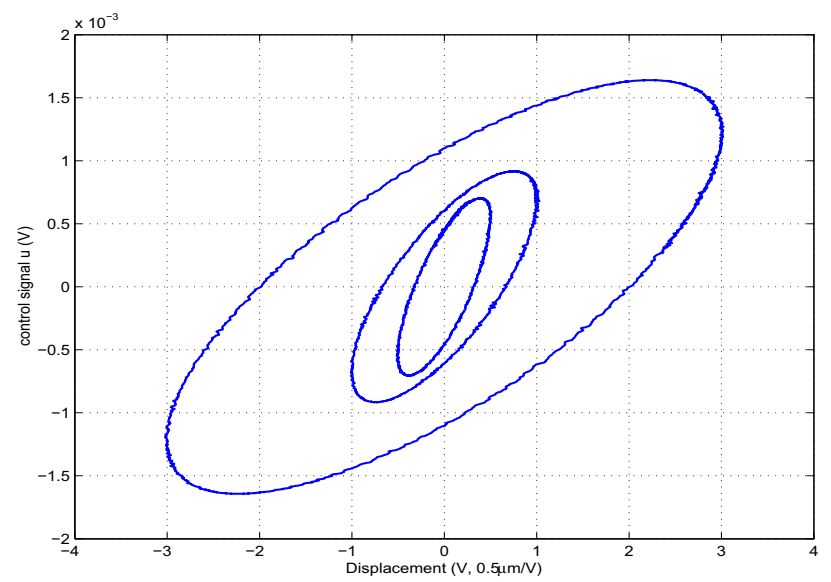

Fig. 3. Control signal $u$ versus position $x$.

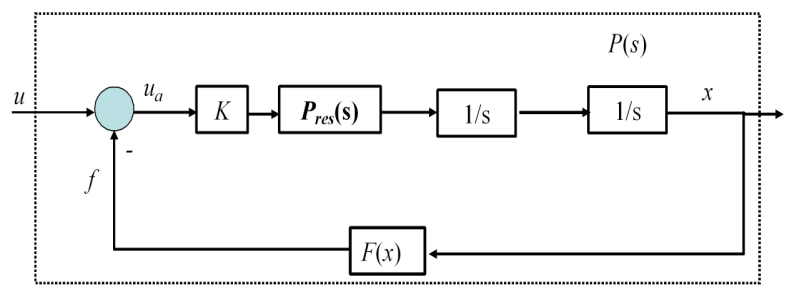

Fig. 4. VCM actuator modeling with friction nonlinearity model $F(x)$.

In what follows, we shall find $F(x)$ to model the relationship between $f$ and $x$. An operator based method to approximate hysteresis will be introduced theoretically and then applied to model the hysteresis of $f$ and $x$ as shown in Fig. 7. 


\section{OPERATOR BASED FRICTION MODELING}

In this section, a mathematical model will be used to closely describe the hysteresis behaviour, then in the next section, the model is used to linearize the actuator response. Among existing hysteresis models in literature, the Prandtl model is less complex and more attractive in real-time applications. The elementary operator in Prandtl hysteresis model [12] is a rate-independent backlash or linear play operator, defined by $p_{r}\left(\pi_{0}, x(t)\right)$, where $x(t)$ is actuator response and $\pi_{0} \in R$ is usually initialized to 0 . Hysteresis nonlinearity can be modeled by a linearly weighted superposition of many backlash operators with different threshold $r>0$ and weight values $w_{b}$, i.e.,

$$
F_{h}(x(t))=\int_{0}^{\infty} w_{b}(r) p_{r}\left[\pi_{0}, x(t)\right] d r
$$

where the weight $w_{b}$ defines the ratio of the backlash operator, as seen in Fig. 5. In order to have an accurate mathematical model for the hysteresis, the creep model proposed in [11] is also incorporated. Hence we consdier the operator model given by

$$
\begin{aligned}
F(x(t))= & a x(t)+\int_{0}^{\infty} w_{b}(r) p_{r}\left[\pi_{0}, x(t)\right] d r \\
& +\int_{0}^{\infty} w_{c}(\lambda) l_{\lambda}\left[\xi_{0}, x(t)\right] d \lambda
\end{aligned}
$$

where $t \in[0, T], a, w_{b}(r)$ and $w_{c}(\lambda)$ are paramteres to be determined, $p_{r}$ and $l_{\lambda}$ are the elementary hysteresis and linear creep operators, and defined as follows.

The elementary hysteresis operator $p_{r}$ with threshold $r$ is defined as the solution operator $p_{r}\left[\pi_{0}, x(t)\right]=z_{r}(t)$ of the rate independent hybrid differential equation

$$
\dot{z}_{r}(t)=\left\{\begin{array}{l}
\dot{x}(t), \quad \text { if } x(t)=z_{r}(t)-r \\
0, \quad \text { if } z_{r}(t)-r<x(t)<z_{r}(t)+r \\
\dot{x}(t) \quad \text { if } x(t)=z_{r}(t)+r
\end{array}\right.
$$

with the initial value equation

$$
z_{r}(0)=\max \left\{x(0)-r, \min \left\{x(0)+r, \pi_{0}(r)\right\}\right\} .
$$

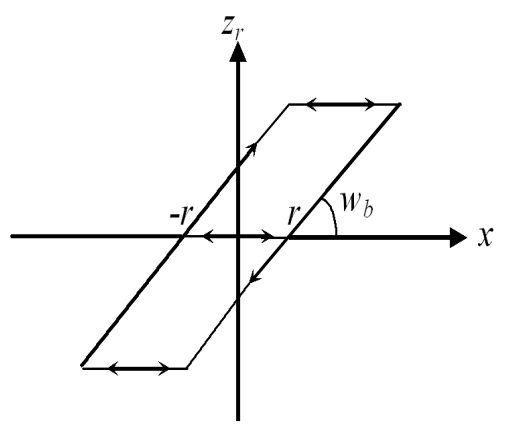

Fig. 5. The operator $z_{r}$ versus $x$.
Define the linear creep operator $l_{\lambda}$ with $\lambda>0$ as the solution operator $l_{\lambda}\left[\xi_{0}, x(t)\right]=z_{\lambda}(t)$ of the differential equation

$$
\frac{1}{\lambda} \dot{z}_{\lambda}(t)+z_{\lambda}(t)=x(t)
$$

with the inital value equation

$$
z_{\lambda}(0)=\xi_{0}(\lambda)
$$

The explicit integral formula for the linear creep operator $l_{\lambda}$ is as follows:

$$
l_{\lambda}\left[\xi_{0}, x(t)\right]=e^{-\lambda t} \xi_{0}(\lambda)+\lambda \int_{0}^{t} e^{\lambda(\tau-t)} x(\tau) d \tau .
$$

For numerical implementation of the operator-based modeling, the discrete-time model $F(x(k))$ of the operator $F(x(t))$ in (4) is developed as follows.

$$
\begin{aligned}
& F(x(k))= \\
& \quad a x(k)+\sum_{i=1}^{n} w_{b i} p_{r_{i}}\left[\pi_{0}, x(k)\right]+\sum_{j=1}^{m} w_{c j} l_{\lambda_{j}}\left[\xi_{0}, x(k)\right]
\end{aligned}
$$

where

1) the output sequence of the discrete hysteresis operator is calculated by

$$
p_{r_{i}}\left[\pi_{0}, x(k)\right]=z_{r_{i}}(k)
$$

$$
\begin{aligned}
& z_{r_{i}}(k)= \\
& \left\{\begin{array}{l}
x(k)+r_{i} \text { if } z_{r_{i}}(k-1)-r_{i} \geq x(k) \\
z_{r_{i}}(k-1) \text { if } z_{r_{i}}(k-1)-r_{i}<x(k)<z_{r_{i}}(k-1)+r_{i} \\
x(k)-r_{i} \text { if } z_{r_{i}}(k-1)+r_{i} \leq x(k)
\end{array}\right.
\end{aligned}
$$

with the initial value $z_{r_{i}}(0)=\max \left\{x(0)-r_{i}, \min \{x(0)+\right.$ $\left.\left.r_{i}, \pi_{0}\left(r_{i}\right)\right\}\right\}$;

2) the discrete counterpart to the continuous elementary creep operator is given by

$$
l_{\lambda_{j}}\left[\xi_{0}, x(k)\right]=z_{\lambda_{j}}(k)
$$

with

$$
z_{\lambda_{j}}(k+1)=e^{-\lambda_{j} T_{s}} \cdot z_{\lambda_{j}}(k)+\left(1-e^{-\lambda_{j} T_{s}}\right) \cdot x(k)
$$

and the initial value $z_{\lambda_{j}}(0)=\xi_{0}\left(\lambda_{j}\right)$.

\section{FRICTION MODELING AND COMPENSATION FOR THE HDD}

\subsection{Friction modeling}

We consider the hysteresis curve of the friction $f$ versus the displacement $x$ in Fig. 7. Let $f_{e}=F(x(k))$ be the approximated friction, then the approximation error $e=$ $f-f_{e}$. We define the energy gain between the actuator position and the error as

$$
\left\|T_{e x}\right\|_{\infty}=\sqrt{\frac{\sum_{k=1}^{L} e^{T}(k) e(k)}{\sum_{k=1}^{L} x^{T}(k) x(k)}},
$$

where $L$ is the number of data points. 
Denote $w_{\mathbf{b}}=\left(w_{b 1}, w_{b 2}, \cdots, w_{b n}\right), w_{\mathbf{c}}=\left(w_{c 1}, w_{c 2}, \cdots, w_{c m}\right)$, and $\Lambda=(\lambda(1), \lambda(2), \cdots, \lambda(m))$. Our purpose is to find optimal parameters $a, w_{\mathbf{b}}, w_{\mathbf{c}}$, and $\Lambda$ in (8) so that (12) is minimized, and thus a model (8) can be obtained to approximate the friction $f$ with the displacement $x$ as the input.

Note that $\left\|T_{e x}\right\|_{\infty}$ is a function of $a, w_{\mathbf{b}}, w_{\mathbf{c}}$, and $\Lambda$, and is denoted as

$$
\left\|T_{e x}\right\|_{\infty}=\ell\left(a, w_{\mathbf{b}}, w_{\mathbf{c}}, \Lambda\right) .
$$

The Matlab function 'fminsearch' can be used to minimize $\ell\left(a, w_{\mathbf{b}}, w_{\mathbf{c}}, \Lambda\right)$ with respect to $\left(a, w_{\mathbf{b}}, w_{\mathbf{c}}, \Lambda\right)$.

$r_{i}$ of (9) can be chosen as the amplitude of $x$. Since With the chosen peak-to-peak values of $x=0.5 \mathrm{~V}, 1 \mathrm{~V}$ and $3 \mathrm{~V}$, $r_{i}$ are valued as

$$
r_{1}=0.25 ; r_{2}=0.5 ; r_{3}=1.5 \text {. }
$$

With $m=3, n=3$ and the initial values $\pi_{0}=0, \xi_{0}=0$, after 2000 iterations, a minimum error $e$ is achieved and the optimal parameters are obtained as

$$
\begin{aligned}
& a=9.0024 \\
& w_{b 1}=-1.5783 ; w_{b 2}=0.1667 ; w_{b 3}=-0.0655 \\
& w_{c 1}=-0.1431 ; w_{c 2}=-7.3341 ; w_{c 3}=0.4403
\end{aligned}
$$

which gives the minimal $\left\|T_{e x}\right\|_{\infty}=0.08$.

With these parameters, $f_{e}=F(x(k))$ can be calculated from (8). The time traces of $f_{e}$ and $f$ are compared in Fig. 6. It is observed that the time trace from the model (8) can give a close tracking of $f$, and the error $f-f_{e}$ is small. The modeled hysteresis from $x$ to $f_{e}$ is drawn and compared with the measured one in Fig. 7. It is seen that the modeled hysteresis and the measured one are close to each other.

Remark With lower excitation the measurement quality is more affected by the nonlinearity, which can be seen from the frequency response comparison between the model and the measurement data in Fig. 8 where the frequency responses for the case of the magnitude of $0.5 \mathrm{~V}$ do not match as well as the other two cases from $70 \mathrm{~Hz}$ to 150 $\mathrm{Hz}$.

Note that the operator model $f_{e}=F(x(k))$ describes the hysteretic characteristics in Fig. 7 as a mapping between the actuator position $x$ and the friction force $f$. It turns out that the model makes it possible to approximate hysteretic transfer characteristics without resorting much to the underlying physics. This is different from the friction models such as the preload and two-slope model in $[3,5]$. As compared to the model in $[3,5]$, an advantage of our operator based model is that in frequency domain the frequency response of the actuator can also fit well to the measured one, as shown in Fig. 8.

For comparison, we also apply the preload and two slope model [3] for the hysteresis, as shown in Fig. 9. The preload model for velocity $v$ and the two-slope model for position $x$ are given by

$$
f_{v}=k_{v} v+k_{s} \operatorname{sgn}(v),
$$

and

$$
f_{x}=\left\{\begin{array}{l}
k_{a} x,|x|<s_{x} \\
k_{b} x+\left(k_{a}-k_{b}\right) s_{x},|x|>s_{x}
\end{array}\right.
$$

Using the data with the amplitude of $x(t)$ of $0.25 \mathrm{~V}$, we obtain $k_{s}, k_{v}, k_{a}, k_{b}$, and $s_{x}$. A comparison with the measured data in the time domain is shown in Fig. 10 for the amplitude of $x(t)$ of 0.25 and $0.5 \mathrm{~V}$, respectively. It can be observed that the plant input $u$ versus position $x$ fits reasonably well to the measured results in the time domain. However, in the frequency domain, the plant magnitude response for the case of amplitude of $x(t)$ of $0.25 \mathrm{~V}$ plotted as the crossed curve in Fig. 8 deviates much from the measurement results.

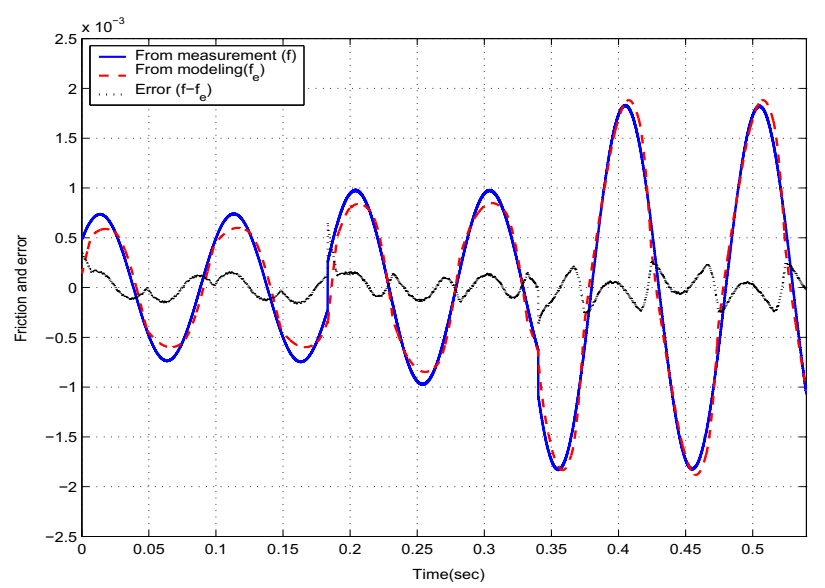

Fig. 6. Measured and modeled friction and error.

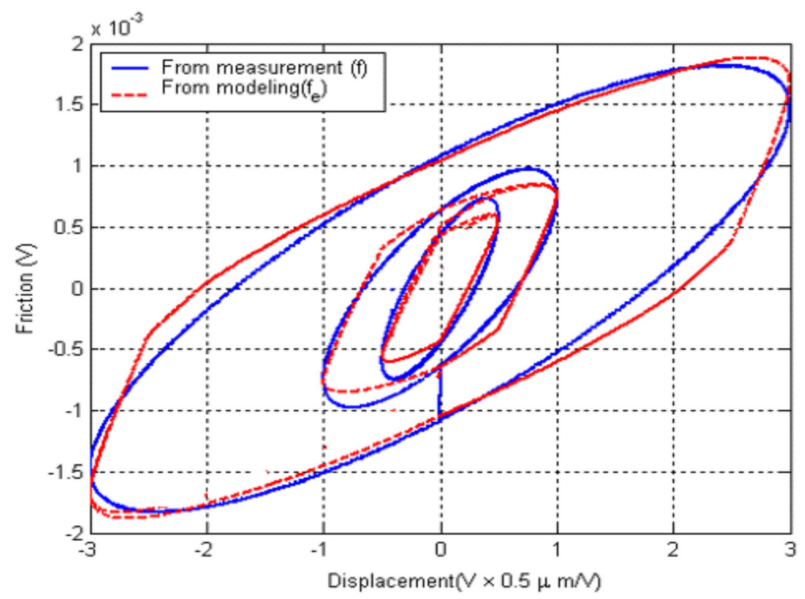

Fig. 7. Friction $f$ versus actuator displacement $x$.

\subsection{Friction compensation}

With the model $f_{e}=F(x(k))$ identified in the previous subsection, the friction can be compensated by injecting the friction force $f_{e}$ into the plant, as seen in Fig. 11. A sinusoidal signal of $50 \mathrm{~Hz}$ and $1 \mathrm{~V}$ amplitude is injected as the reference. The input $u$ versus the actuator displacement $x$ is compared for the cases with and without the nonlinear compensation in Fig. 12. It is clearly evident that with the nonlinear compensation the relationship between $u$ and $x$ is linearized very well. In the frequency domain, 

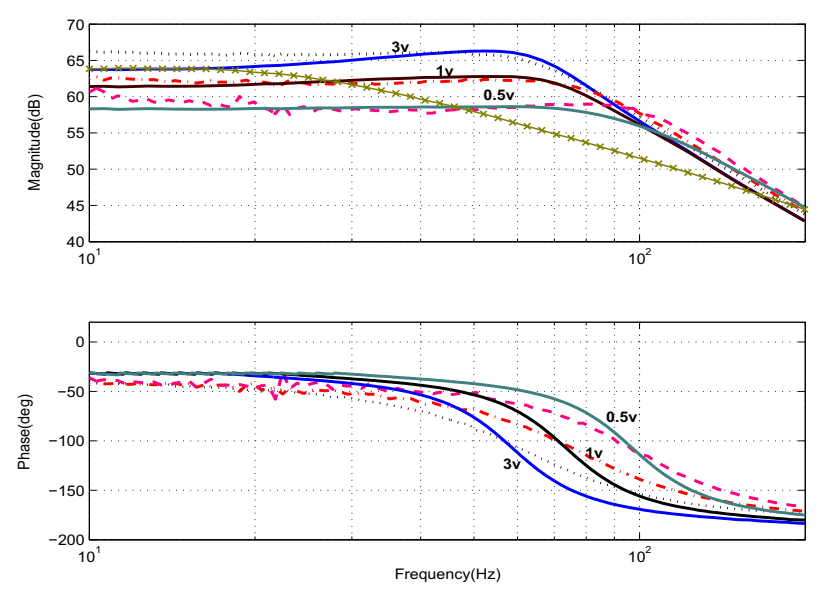

Fig. 8. Actuator frequency response for sinusoidal reference of $0.5,1$ and $3 \mathrm{~V}$ amplitude (Solid curves: the modeled; dashed, dotted, and dash-dotted curves: the measured).

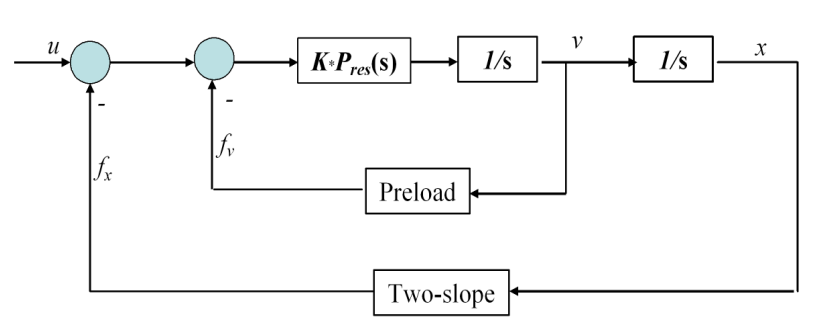

Fig. 9. Preload and two-slope model for friction modeling.

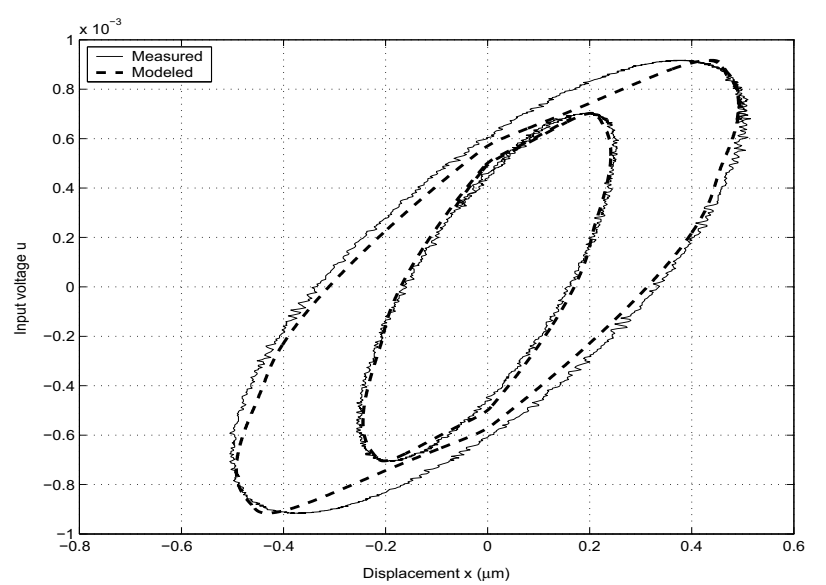

Fig. 10. Plant input voltage $u$ versus displacement $x$.

the actuator frequency responses before and after the compensation are measured with swept sinewave via DSA and shown in Fig. 13. It can be seen that the compensated magnitude and phase responses approach those of the pure double integrator much more closely than those before compensation.

With the friction compensation, the VCM actuator frequency responses are measured with different sinusoidal reference amplitudes and plotted in Fig. 14, where the straight smooth lines are for the pure double integrator. It is seen that the linearization effect becomes better when the reference amplitude is higher than $1 \mathrm{~V}(0.5 \mu \mathrm{m} / \mathrm{V})$, and it is not so satisfactory for $0.25 \mathrm{~V}$. Although the friction model is obtained on the basis of the measurement for 0.5 , 1 , and $3 \mathrm{~V}$ displacement amplitudes, the compensation based on the obtained model is able to achieve good linearization effect for any displacement ranging from 0.5 $\mathrm{V}$ and above.

Correspondingly the simulated and measured error rejection functions (or sensitivity functions) are shown in Fig. 15. We observe that with the compensation the magnitude at $10 \mathrm{~Hz}$ is reduced by around $20 \mathrm{~dB}$ due to the increased open-loop gain at low frequency as seen in Fig. 14. The slightly lower magnitude from 60 to $100 \mathrm{~Hz}$ before compensation, corresponding to the higher magnitude of the original actuator model from 60 to $100 \mathrm{~Hz}$ in Fig. 13, is caused by the nonlinearity of the original actuator.

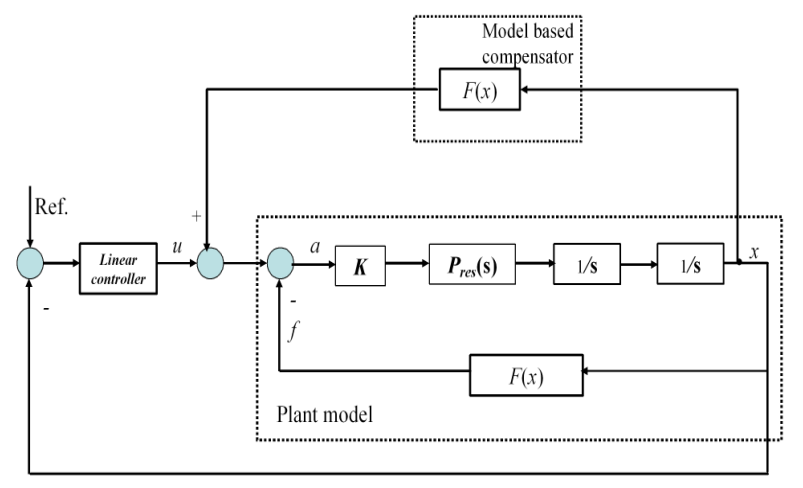

Fig. 11. Friction compensation for the VCM actuator.

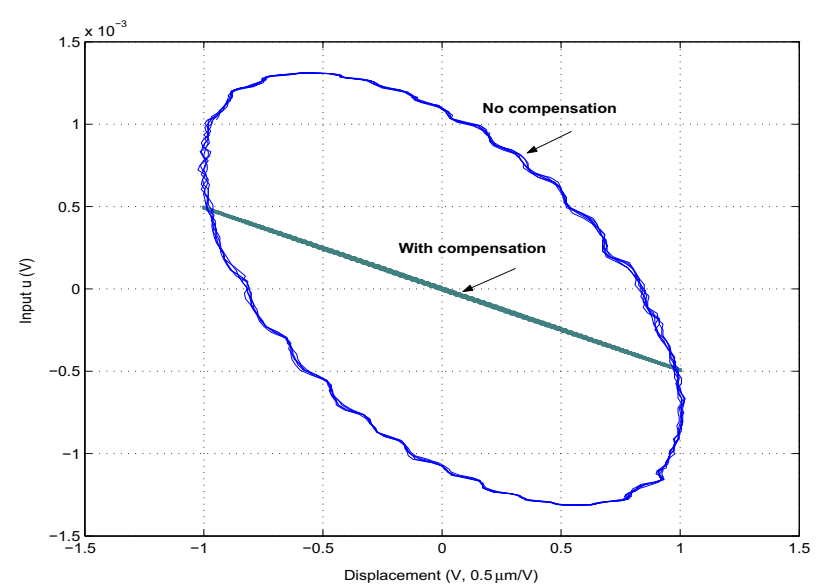

Fig. 12. Input $u$ versus displacement $x$ with and without compensation.

\section{CONCLUSION}

In this paper the Prandtl operator based model has been used to model the pivot friction of VCM actuator for a 1.8inch small disk drive under the realistic conditions of rotating disk and flying slider. Optimal model parameters have been obtained by minimizing the energy gain between the actuator position and the modeling error. The obtained model matches well the measured model not only in time domain, but also in frequency domain. Subsequently, the nonlinear friction has been compensated by injecting the 

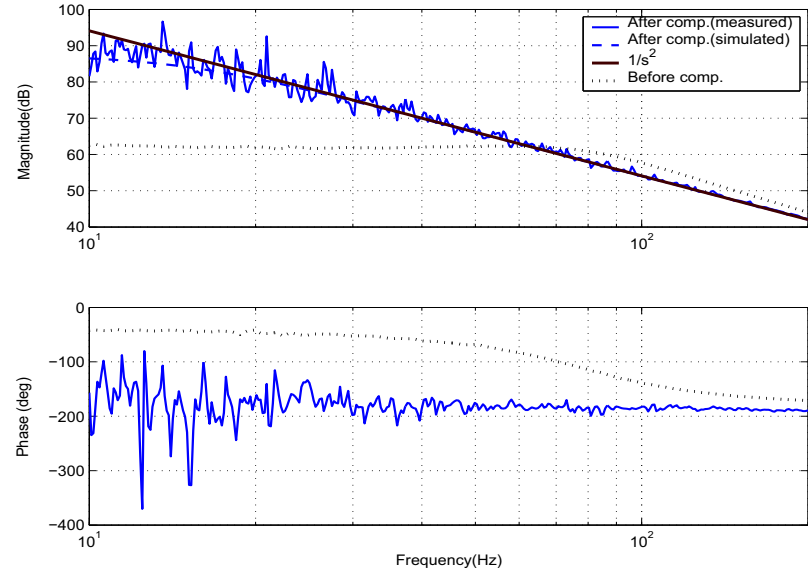

Fig. 13. Actuator frequency responses with and without friction compensation.
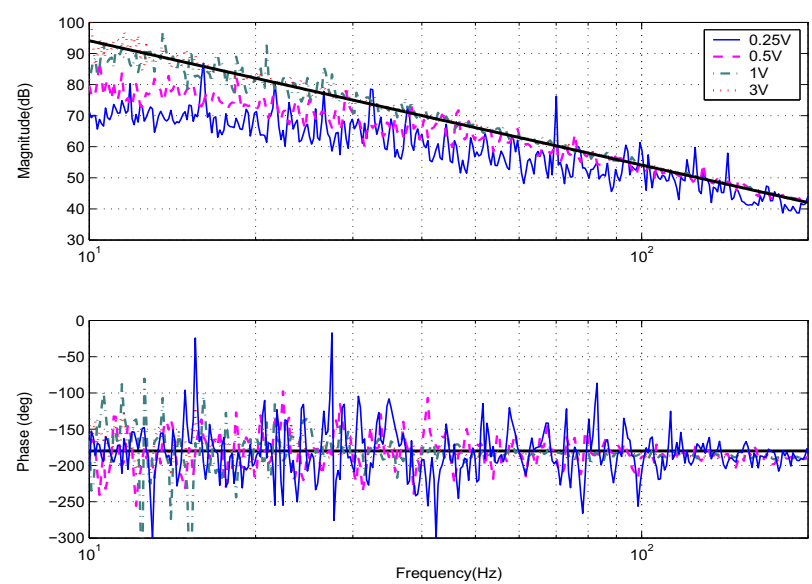

Fig. 14. Actuator frequency responses with friction compensation for different displacements in voltage with $0.5 \mu \mathrm{m} / \mathrm{V}$ (Straight smooth lines are for the pure double integrator).

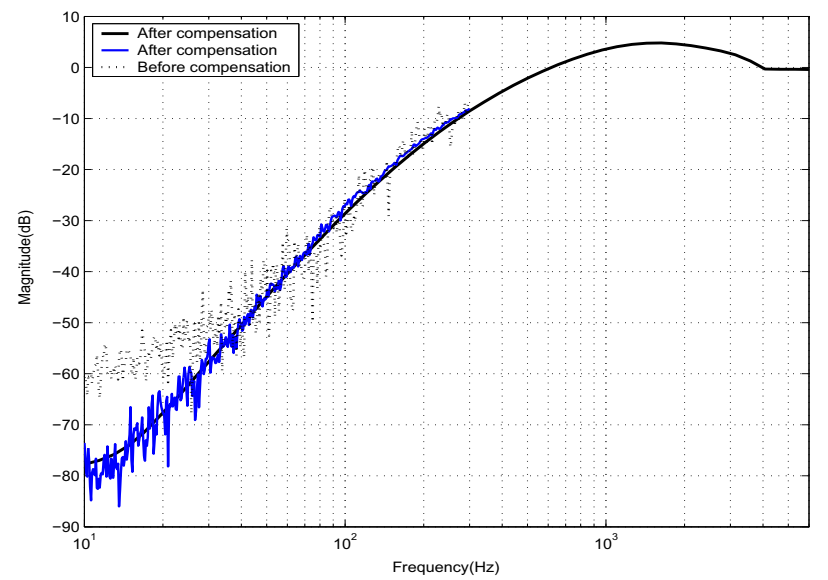

Fig. 15. Sensitivity functions with and without friction compensation. modeled friction force into the VCM actuator. With the model based compensation, the linearization effect for the VCM actuator has been verified via the measurement of the hysteresis in time domain and the frequency response in frequency domain. The measured error rejection function has shown an increased error rejection capability at low frequency, resulted from the compensation.

\section{REFERENCES}

[1] R. W. Wood, and H. Takano, "Prospects for magnetic recording over the next 10 years", IEEE International Magnetics Conf., San Diego, California, USA, May 812, pp. 98, 2006.

[2] JyhChing Juang and JengShi Chen, "On combining adaptive estimation and robust control for friction compensation", Proc. of the 5th World Congress on Intelligent Control and Automation, 2004.

[3] D. Abramovitch, F. Wang, and G. Franklin, "Disk drive pivot nonlinearity modeling Part 1: Frequency domain", Proc. of American Control Conf., Baltimore, Maryland, USA, June 1994, pp. 2600-2603.

[4] T. Yan and R. Lin, "Experimental modeling and compensation of pivot nonlinearity in Hard Disk Drives", IEEE Trans. on Magn., vol. 39, no. 2, Mar. 2003.

[5] F. Wang, T. Hurst, D. Abramovitch, G. Franklin, Disk drive pivot nonlinearity modeling part II: Time domain, Proc. of American Control Conf., Baltimore, Maryland, USA, June, 1994, pp. 2604-2607.

[6] J. Ishikawa, M. Tomizuka, A Novel Add-On Compensator for Cancellation of Pivot nonlinearities in Hard Disk Drives, IEEE Trans. on Magnetics, vol. 34, No. 4, 1998.

[7] M. Kemal Ciliz and Masayoshi Tomizuka, Modeling and Compensation of Frictional Uncertainties in Motion Control: A Neural network Based Approach, Proc. of the American Control Conf., 1995.

[8] T. Huang, Y. Ding, S. Weerasooriya, T. S. Low, Disk Drive Pivot Nonlinearity Modeling and Compensation through Fuzzy Logic, IEEE Trans. on Magnetics, vol. 34, No. 1, 1998.

[9] Y. Tanaka, "Fundamental features of perpendicular magnetic recording and the design consideration for future portable HDD integration," IEEE International Magnetics Conf., Nagoya, Japan, pp. 163-164, 4-8 April 2005.

[10] K. Peng, B. Chen, G. Cheng, T. H. Lee, Modeling and compensation of nonlinearities and friction in a micro Hard Disk Drive servo system with nonlinear feedback control, IEEE Trans. on Control Systems Technology, vol. 13, no. 5, Sept. 2005, pp. 708-721.

[11] P. Krejci and K. Kuhnen, "Inverse control of systems with hysteresis and creep", IEE Proc.- Control Theory and Application, vol. 148, no. 3, May 2001, pp. 185-192.

[12] M. Brokate, and J. Sprekels, "Hysteresis and Phase Transitions", Springer-Verlag, Berlin-Heidelberg-New York, 1996. 